\section{OP0097 DEVELOPMENT OF NEW INTERNATIONAL CLASSIFICATION CRITERIA FOR ANTIPHOSPHOLIPID SYNDROME: PHASE III CASE COLLECTION RESULTS}

M. Barbhaiya ${ }^{1}$, D. Erkan ${ }^{1}$, Y. Ahmadzadeh ${ }^{1}$, K. Costenbader ${ }^{2}$, R. Naden ${ }^{3}$, S. Zuily ${ }^{4}$ on behalf of the New APS Classification Criteria Collaborators and Phase III Case Collectors. ${ }^{1}$ Hospital for Special Surgery, Weill Cornell Medicine, New York, United States of America; ${ }^{2}$ Brigham and Women's Hospital, Harvard Medical, Boston, United States of America; ${ }^{3}$ Auckland City Hospital, Auckland, New Zealand; ${ }^{4}$ Nancy Academic Hospital, Nancy, France

Background: An international multi-disciplinary effort is underway to develop rigorous, new, consensus- and evidence-based classification criteria for Antiphospholipid Syndrome (APS). The methodological approach includes four phases; we have previously presented phases I and II (item generation and reduction), which resulted in 27 candidate criteria $^{1}$ organized in laboratory and clinical domains.

Objectives: Phase III (item weighting/threshold identification) is currently underway; here we report initial Phase III case collection results.

Methods: We used REDCap, a secure data system, for Phase III international case collection. The candidate criteria of 27 items at the end of Phase II was represented in a standardized case collection form. We asked international physicians interested in APS to provide and rate cases using a Likert scale $(+3$ to -3 : highly likely to highly unlikely to be APS). Cases with higher scores $(+2$ or +3$)$ were categorized as "highly likely" APS, whereas lower scores (+1 to -3$)$ were categorized as "equivocal or unlikely" APS.

Results: We collected 314 potential APS cases (mean age $43.8 \pm 14.4$ years; $79 \%$ female; and $77 \%$ white) between 6/2019-8/2019 from 17 sites in Europe $(47 \%)$, North America (41\%), and South America (11\%). Majority of cases were potential primary APS (64\%); 137 were rated as "highly likely" and 177 as "equivocal or unlikely" APS. Lupus anticoagulant, anti $\beta_{2}$ glycoprotein-I antibody IgG/M, anticardiolipin antibody $\mathrm{lgG}$, arterial thrombosis, venous thromboembolism, microvascular disease, fetal loss between 16-34 weeks, severe preeclampsia, severe placental insufficiency, cardiac valve disease, and low platelet count occurred with higher frequency in the APS cases categorized as "highly likely" (Table).

Conclusion: International collection of cases spanning the spectrum of "highly likely" to "equivocal or unlikely" APS provide "real world" assessment of patients being referred for APS evaluation. In next steps, proposed candidate criteria will be further refined, organized, and weighted, and a preliminary threshold for APS classification will be determined.

Table. Characteristics of Potential APS Cases Categorized by Physician Assessment

\begin{tabular}{cccc}
\hline Candidate Relative Criteria, $n(\%)$ & Total & Highly Likely & Equivocal \\
& $(n=314)$ & APS & or Unlikely \\
& & $(n=137)$ & APS \\
$(n=177)$
\end{tabular}

\section{LABORATORY}

Lupus Anticoagulant Test

Anticardiolipin Antibody

- $\lg G$

- IgM

Anti $\beta_{2}$ glycoprotein-I Antibody

- $\lg G$

- IgM

CLINICAL

Macrovascula

- Superficial Vein Thrombosis

- Venous Thromboembolism

- Transient Ischemic Attack

- Arterial Thrombosis

Microvascular ${ }^{\star}$

Obstetric $^{\star \star}$

- Embryonic Loss (<10 weeks [w])

- Fetal Loss (10w-<16w)

- Fetal Loss $(16 w-34 w)$

- Severe Preeclampsia $(<34 \mathrm{w})$

- Severe Placental Insufficiency ( $<34 w)$

Cardiac Valve Disease ${ }^{\star \star \star}$

Thrombocytopenia $<150 \mathrm{G} / \mathrm{L}$

$\begin{array}{lcc}208(66) & 117(85) & 91(51) \\ 170(54) & 90(66) & 80(45) \\ 138(44) & 61(45) & 77(44) \\ & & \\ 99(32) & 66(48) & 33(19) \\ 79(25) & 47(34) & 32(18) \\ & & \\ 15(5) & 4(3) & 11(6) \\ 95(30) & 63(46) & 32(18) \\ 27(9) & 13(10) & 14(8) \\ 82(26) & 58(42) & 24(14) \\ 86(27) & 62(45) & 24(14) \\ & & \\ 85(45) & 34(42) & 51(48) \\ 20(11) & 9(11) & 11(10) \\ 42(22) & 32(40) & 19(18) \\ 27(14) & 18(22) & 9(8) \\ 22(12) & 13(16) & 9(8) \\ 32(10) & 21(15) & 11(6) \\ 72(23) & 43(31) & 29(16)\end{array}$

*Livedo racemosa, livedoid vasculopathy, adrenal hemorrhage, acute ischemic enceophalopathy, cardiac microvascular disease, pulmonary hemorrhage, acute and/or chronic antiphospholipid-related nephropathy; ${ }^{* \star}$ Total number of patients ever pregnant: 188 (highly likely APS: 81; unlikely APS: 107 ) ${ }^{\star \star \star}$ Valve thickening and/or vegetation.

\section{References:}

[1] Barbhaiya M et al. Phase II Results [abstract]. Arthritis Rheumatol. 2019;71 (suppl 10).

Acknowledgments: The project is supported by ACR/EULAR

Disclosure of Interests: Medha Barbhaiya: None declared, Doruk Erkan: None declared, Yasaman Ahmadzadeh: None declared, Karen Costenbader Grant/ research support from: Merck, GSK, Consultant of: Merck, GSK, Lily, Astra Zeneca, Janssen, Raymond Naden: None declared, Stéphane Zuily: None declared

DOI: 10.1136/annrheumdis-2020-eular.5294

\begin{tabular}{|l|l}
\hline OP0098 & TRAJECTORIES OF GROWTH IN OFFSPRING \\
FROM MOTHERS WITH PREVALENT OR PRE- \\
SYSTEMIC LUPUS ERYTHEMATOSUS: A \\
NATIONWIDE POPULATION-BASED STUDY \\
IN KOREA
\end{tabular}

I. A. Choi ${ }^{1}$, J. H. Kim', S. A. Yoon ${ }^{2}$, S. H. Chang ${ }^{3}$, H. J. Kim ${ }^{4}$, H. S. Ahn .

${ }^{1}$ Chungbuk National University Hospital, Division of Rheumatology, Department of Internal Medicine, Cheongju, Korea, Rep. of (South Korea); ${ }^{2}$ Chungbuk National University Hospital, Department of Pediatrics, Cheongju, Korea, Rep. of (South Korea); ${ }^{3}$ Soonchunhyang University, Division of Rheumatology, Department of Internal Medicine, Cheonan, Korea, Rep. of (South Korea); ${ }^{4}$ College of Medicine, Korea University, Institute for Evidence-based Medicine, Cochrane Korea, Department of Preventive Medicine, Seoul, Korea, Rep. of (South Korea)

Background: Systemic lupus erythematosus (SLE) is an autoimmune disease that primarily affects women of childbearing age and is known to have poor maternal and fetal outcomes associated with pregnancy.

Objectives: We planned on the long-term follow-up studies to find out whether SLE offspring with low birth weight would properly catch up in future childhood life.

Methods: Korean National Health Insurance (NHI) service is a mandatory, single public health insurance system that covers more than 50,000,000 Korean population. It consists of inpatient and outpatient diagnosis and treatment data for the purpose of reimbursement. Rare and Intractable Diseases (RID) registration system is a copayment reduction scheme which provides financial support to patients with certain rare disease, include SLE. National Health Screening Program for Infants and Children (NSHPIC) is a population screening system for children aged 4 to 71 months, requiring 7 visits to participating clinic at pre-specified times.

Among the children who were born between 2008 and 2013, we identified those who completed the first (between 4-6th month), second (between 9-12th month) and either sixth or seventh examination (between 54-65th month and 66-71th month, respectively) of NSHPIC. By linking maternal and offspring healthcare data through their unique personal identification numbers, we constructed a mother-child database to track the growth of the child. Among their mothers, we could identify SLE patients who had given live singleton births using RID database (V136) and the international classification of disease code (M32.x) for SLE. Therefore, we were able to follow birth weight and subsequent growth of offspring according to their mother's disease status.

Results: We could identify 1,007 offspring from SLE mothers and 793,537 control from non-SLE mothers. Offspring from SLE mothers showed the higher risk of low birth weight defined as less than $2500 \mathrm{~g}$ [hazard ratio $(\mathrm{HR})$ $4.79,95 \%$ confidence interval $(\mathrm{Cl}) 4.08-5.63$ ]. In terms of subgroups analysis according to the time of diagnosis, the risk of low birth weight was the highest in offspring from those who diagnosed SLE during pregnancy (HR $8.22,95 \% \mathrm{Cl} 3.18-21.26)$. In addition, the risk of low birthweight increased not only in offspring from already diagnosed SLE before pregnancy (HR 5.63 $95 \% \mathrm{Cl} 4.41-7.19)$, but also in offspring from those who diagnosed with SLE after delivery (pre-SLE) compared to general population (HR 2.03, 95\% Cl 1.33-3.09).

Subsequent growth failure defined as less than 3 percentile was more prevalent in SLE offspring compared to general population at 4-6 month (HR 3.28, 95\% Cl 2.22-4.77). However, at 6-9 months and 54-71 months, the gap was reduced, showing no statistical difference with the general population (HR 1.22, 95\% Cl 0.73-2.03 and $\mathrm{HR} 1.26,95 \% \mathrm{Cl} 0.88-1.80$, respectively).

Among SLE offspring with low birth weight $(<2500 \mathrm{~g}), 89.34 \%$ showed the body weight of 3 percentile or more and $75.84 \%$ showed the body weight 10 percentile or more at 4-6 month. The proportion of children who catch up afterwards has not increased since then, as the proportion of children who 
were more than 10 percentile was $81.46 \%$ at $6-9$ months and $80.34 \%$ at $54-71$ months.

Conclusion: The offspring of SLE patients were more likely to have low birthweight compared to general population, but majority of them showed the catch up growth at 4-6 month of age. The risk of low birthweight was especially high in the offspring from mothers who diagnosed SLE during pregnancy.

Disclosure of Interests: None declared

DOI: 10.1136/annrheumdis-2020-eular.4002

\section{New technologies in basic research}

\begin{tabular}{|l|l}
\hline OP0099 & FUNCTIONAL MAPPING OF SYNOVIAL \\
& FIBROBLAST POPULATIONS IN HEALTH AND \\
& ARTHRITIC DISEASE: INSIGHTS INTO THE \\
& PATHOGENIC REMODELING OF SYNOVIAL \\
& MICROENVIRONMENT
\end{tabular}

D. Konstantopoulos ${ }^{1}$, C. Tzaferis ${ }^{2}$, M. Sakkou ${ }^{2}$, A. Liakos3, M. Lavigne3, D. Karamitros ${ }^{2}$, M. Fousteri ${ }^{1}$, M. Armaka ${ }^{1}$, G. Kollias ${ }^{2,4}$. ${ }^{1}$ B. S. R.C. "Alexander Fleming", Institute of Fundamental Biomedical Research, Vari, Greece; ${ }^{2}$ B.S.R.C. "Alexander Fleming", Institute of Bioinnovation, Vari, Greece; ${ }^{1}$ B.S.R.C. "Alexander Fleming", Institute of Fundamental Biomedical Research, Vari, Greece; ${ }^{4}$ Medical School of Athens, Department of Physiology, Athens, Greece

Background: Our previous studies highlighted the fundamental in vivo role of synovial fibroblasts (SFs) in TNF-mediated murine chronic arthritis ${ }^{1,2}$ and recent findings identified different SF identities based on their transcriptomic profiles with distinct contributions in acute, autoimmunity-based, murine arthritis ${ }^{3}$.

Objectives: In this study, we focus on delineating the map of SF subpopulations in healthy joint and in the course of arthritic disease and the underlying regulatory networks functioning towards pathogenicity.

Methods: Sorted single cell suspensions (CD45-, Pdpl+) and their fragmented nuclei from synovial joints of WT, early and late arthritic hTNFtg mice were processed for scRNAseq and scATAC employing a droplet-based technology (10x Genomics). To define the transcriptional and epigenetic signatures originating from the two different assays, we developed an integrative analysis pipeline based on the Seurat software package (v3.1). Meta-analysis of previously reported data of $\mathrm{K} / \mathrm{B} \times \mathrm{N}$ serum transfer of arthritis was employed to define commonalities and differences in SF subsets among murine modelled disease.

Results: The transition from healthy to chronically affected synovial microenvironment (SME) due to overexpression of hTNF is characterized by a dynamic transformation of SF clusters. The Lining arthritic Thy $1^{\text {low }}$ synovial layer (L-SFs) is hyper-populated while Sub-Lining Thy $1^{\text {high }}$ SF clusters (SL-SFs) are remodeled towards catabolic and inflammatory phenotypes compared to naïve SF organization pattern. Interestingly, trajectory analysis revealed that the SL clusters, which normally exhibit a gradual developmental-like process towards different profiles, differentially change during disease. We identified that the previously reported proliferating SL cluster is absent in healthy synovium, dominates mainly in early stages of chronic arthritis and it is closely related to the L-SFs. Mapping of the gene regulatory networks by RNAseq was supported by scATAC analysis. Similarly, meta-analysis of SF profiles derived from naïve and the $\mathrm{K} / \mathrm{BxN}$-serum-treated mice showed significant differences, possibly reflecting the phenotypes of the two established models of arthritis.

Conclusion: Our approach unravels for the first time the regulatory heterogeneity and gene expression profiling of SF subpopulations in normal synovium, and reveals deep biological insights of the functional re-organization of SME during development of disease. It further identifies the common and divergent features of the different subtypes of murine arthritis that may well reflect the diversity of RA subtypes and the response to therapies.

References:

[1] Armaka et al; J Exp Med (2008): 205(2):331-7.

[2] Armaka et al; Nat Commun (2018): 9(1):618.

[3] Croft et al; Nature (2019): 570(7760):246-251.

Disclosure of Interests: None declared

DOI: 10.1136/annrheumdis-2020-eular.1839

\section{OP0100 MOLECULAR PROFILING OF PERIPHERAL IMMUNE CELL SUBSETS IN PATIENTS WITH RHEUMATOID ARTHRITIS}

P. C. Taylor ${ }^{1}$, J. Liü ${ }^{2}$, L. Zhuo ${ }^{2}$, Y. Tian², T. Snyder ${ }^{3}$, C. Kim ${ }^{3}$, P. Kheradpour ${ }^{3}$, K. Drake ${ }^{3}$, S. Kim ${ }^{2}$, R. E. Hawtin ${ }^{2}{ }^{1}$ University of Oxford, Botnar Research Centre, Nuffield Department of Orthopaedics, Rheumatology and Musculoskeletal Sciences, Oxford, United Kingdom; ${ }^{2}$ Gilead Sciences Inc., Foster City, United States of America; ${ }^{3}$ Verily Life Sciences, South San Francisco, United States of America

Background: Rheumatoid arthritis (RA) is a chronic systemic autoimmune disease that affects $1 \%$ of the world's population. Several key biological functions are dysregulated in RA, manifesting clinically as pain, fatigue, and synovitis, with articular destruction, organ-based comorbidities, and functional decline. Defining immune dysregulation in the peripheral blood of patients (pts) with RA will help inform future work to assess the extent to which immune homeostasis can be therapeutically achieved for these pts.

Objectives: To identify baseline molecular characteristics of the peripheral immune system, at the level of individual immune cell subsets, in pts with RA recruited to clinical trials of the oral, selective Janus kinase 1 (JAK1) inhibitor, filgotinib.

Methods: Peripheral blood mononuclear cells (PBMC) were collected from 324 pts with moderate to severely active RA, who had an inadequate response to methotrexate ([MTX], FINCH-1; NCT02889796; $n=109$ ) or who were MTX naïve (FINCH-3; NCT02886728; $n=215)$. PBMC were also collected from 50 demographically matched healthy volunteers (HV). The Immune Profiler platform was used to sort PBMC into 24 immune cell subsets, then quantify their gene expression and chromatin accessibility using RNA-seq and the assay for transposase-accessible chromatin with high-throughput sequencing (ATACseq), respectively. Differentially expressed genes (DEGs) and differentially accessible regions (DARs) were identified among immune cell subsets from pts with RA versus HV. Gene set signature scores of Molecular Signatures Database hallmark pathways were calculated using single sample gene set enrichment analysis (SSGSEA) to examine differences in pathway activity between groups.

Results: A total of 14,500 sequencing datasets were generated from the pt and HV immune cell subsets. Among these, over 26,000 DEGs and 220,000 DARs were identified in RA versus HV (false discovery rate <0.05) across the 24 immune cell subsets. DEGs were identified in all immune cell subsets tested and were most pronounced in natural killer (NK) subsets; most DARs were detected in myeloid and NK subsets. SSGSEA revealed differential pathway signaling in RA versus HV across multiple functions at the immune cell subset level. Myeloid subsets from pts with RA often showed elevated pathway activities versus HV whereas B, T and NK subsets showed a general decrease. In particular, monocyte populations from pts with RA versus HV had elevated pathway activities involved in inflammatory response and interleukin-6/Janus kinase/signal transducer and activator of transcription 3 signaling. The B, T and NK subsets showed a general decrease in tumor necrosis factor-a signaling; conversely, monocyte subsets showed an increase. Prior MTX exposure did not have a notable impact on the detected molecular profile.

Conclusion: Differences in gene expression, hallmark pathway activity, and chromatin accessibility were identified in RA versus $\mathrm{HV}$ at the immune cell subset level. Significant contributions to differences in chromatin accessibility identified in the myeloid and NK cell populations suggest that there are more active regulatory sequences in these cell types that are associated with RA. Further investigations based on these findings may increase understanding of the immune regulatory paradigm in the context of RA.

Acknowledgments: This study was funded by Gilead Sciences, Inc. Editoria support was provided by Fishawack Communications Inc and funded by Gilead Sciences, Inc.

Disclosure of Interests: Peter C. Taylor Grant/research support from: Celgene, Eli Lilly and Company, Galapagos, and Gilead, Consultant of: AbbVie, Biogen, Eli Lilly and Company, Fresenius, Galapagos, Gilead, GlaxoSmithKline, Janssen, Nordic Pharma, Pfizer Roche, and UCB, Jinfeng Liu Shareholder of: Gilead Sciences Inc., Roche, Employee of: Gilead Sciences Inc., Luting Zhuo Employee of: Gilead Sciences Inc., Yuan Tian Employee of: Gilead Sciences Inc., Thomas Snyder Employee of: Verily Life Sciences, Charlie Kim Employee of: Verily Life Sciences, Pouya Kheradpour Employee of: Verily Life Sciences, Kat Drake Employee of: Verily Life Sciences, Sam Kim Shareholder of: Gilead Sciences Inc., Employee of: Gilead Sciences Inc., Rachael E. Hawtin Shareholder of: Gilead Sciences Inc., Employee of: Gilead Sciences Inc. DOI: 10.1136/annrheumdis-2020-eular.3967 